\title{
¿FUE DARWIN EL «NEWTON DE LA BRIZNA DE HIERBA»? LA HERENCIA DE KANT EN LA TEORÍA DARWINISTA DE LA EVOLUCIÓN
}

\section{WAS NEWTON THE «DARWIN OF A BLADE OF GRASS»? KANT'S LEGACY IN THE DARWINIAN THEORY OF EVOLUTION}

\author{
Laura NUÑO DE LA RosA* \\ Departamento de Filosofía I (Metafísica y Teoría del Conocimiento). \\ Universidad Complutense de Madrid. \\ IHPST. Universidad París 1-Sorbona.
}

Arantza ETXEBERRIA**

Departamento de Lógica y Filosofía de la Ciencia. Euskal Herriko Unibertsitatea UPV/EHU

RESUMEN: La crítica kantiana legó una doble herencia a la biología decimonónica: su noción de ciencia basada en el mecanicismo newtoniano configuró epistemológicamente la teoría de la evolución darwinista, mientras que su comprensión de los organismos se tradujo en una morfología teleológica. En este artículo planteamos dos cuestiones en torno la relación entre las ideas de Kant y Darwin: 1) si Kant habría considerado a Darwin el Newton de la biología, a lo que, con matices, respondemos afirmativamente; 2) si la física newtoniana es hoy suficiente para naturalizar lo orgánico. Nuestra respuesta negativa se fundamenta en las ciencias de la autoorganización y la evo-devo, que permiten plantear la naturalización de la concepción kantiana de los organismos.

* e-mail: lauranrg@gmail.com

** e-mail: arantza.etxeberria@ehu.es 
PALABRAS CLAVE: Teleología, selección natural, árbol de la vida, autoorganización, biología evolutiva del desarrollo.

ABSTRACT: Kantian criticism left a double legacy to the biology of the 19th Century: his notion of science based on Newtonian mechanism motivates and shapes epistemologically Darwin's theory of evolution, whereas his understanding of organisms as natural purposes gave rise to a teleological morphology. In this paper we pose two questions about the relation between Kant's and Darwin's ideas: 1) whether Kant would have considered Darwin to be the Newton of Biology, to which our answer is affirmative; 2) whether Newtonian Physics is today enough to naturalize Biology. Our negative answer, based on self-organization and evo-devo, allows to set out the naturalization of the Kantian approach to organisms.

KEYWORDS: Teleology, natural selection, tree of life, selforganization, Evolutionary Developmental Biology.

Es absurdo esperar que un día pudiera surgir un segundo Newton que hiciera inteligible la producción de una simple hoja de hierba de acuerdo con las leyes de la naturaleza, sin que sus mutuas relaciones no fueran dispuestas por alguna intención.

KANT, Crítica del juicio, $\$ 75$

\section{Introducción}

La filosofía de la biología más reciente ha vuelto su mirada a Kant con el fin de examinar la explicación naturalista de los seres vivos. En la «Crítica del juicio teleológico", el carácter irreductiblemente especial de los organismos se cifra en que, al menos aparentemente y a diferencia de los inanimados, los seres vivos se nos muestran como sistemas autoorganizados. Ahora bien, la negación kantiana de que esta comprensión de lo vivo pueda convertirse en objeto de conocimiento científico anticipa las dificultades a las que habrá de enfrentarse una disciplina que no se ajuste a los esquemas epistemológicos mecanicistas clásicos, y que requiera la invención de nuevas formas de modelización. De hecho, explicar qué tienen de especial los seres vivos sigue siendo uno de los retos filosóficos más desafiantes de la actualidad. 
Históricamente, las posturas positivistas han ensalzado la condena kantiana de la teleología y su destierro al reino de la metafísica. Desde esta perspectiva, se considera que Darwin avanzó en la naturalización de lo orgánico por haber localizado en la selección natural la causa eficiente de la evolución, desconocida en tiempos de Kant. Así, darwinistas como Haeckel ${ }^{1}$ o artífices de la Síntesis Moderna como Mayr (que coinciden en pensar que las causas evolutivas proporcionan el porqué de la fenomenología viviente, mientras que las causas próximas u ontogenéticas sólo aclaran el cómo), suelen presentar la «revolución darwinista» como análoga en biología al giro copernicano y su formalización newtoniana en las ciencias físicas: desde la Grecia Clásica, las especies se concebían tan inmóviles como lo fuera la Tierra para la física de Aristóteles, y Darwin habría jugado el doble papel de Galileo y Newton al imprimir movimiento a los taxones y descubrir en la selección natural la causa motora de su transformación. De este modo, Darwin se habría convertido en ese «Newton de la hoja de hierba» que Kant no fue capaz de imaginar. En general, quienes han visto en Darwin al naturalizador equivalente a Newton creen que la teoría de la evolución basada en la selección natural puede y debe ser el marco integrador de las explicaciones de la fenomenología viviente.

Otros autores, como Roux ${ }^{2}$ y, en general, quienes creen que la comprensión de los organismos está indisolublemente ligada a la comprensión de su generación, han negado ese papel histórico a Darwin, argumentando que para explicar la aparición de las formas y su funcionamiento no es suficiente con postular la existencia de una presión selectiva externa que las favorezca, sino que es necesario dar cuenta de los procesos ontogenéticos que las hacen posible. Por otro lado,

1 Así se refiere Cassirer (1948) a la postura de Haeckel: «[La] eliminación y superación de toda modalidad de concepción teleológica constituye, según Haeckel, la verdadera hazaña emancipatoria llevada acabo por Darwin y sólo comparable con la de Copérnico. (...) El «Newton de la brizna de hierba" cuya posibilidad negaba Kant, convirtióse en una realidad con la persona de Darwin: su teoría de la selección resolvió, realmente, el problema que Kant consideraba insoluble». (p. 200).

2 Así se refiere Cassirer (1948) a la postura de Roux: «En el campo de la fisiología y no de la filogenia espera Roux ver surgir el "Newton de la biología", de cuya posibilidad no duda, aunque diste mucho de creer, con Haeckel, que este Newton sea Darwin: el Newton que él espera logrará construir el movimiento del organismo total partiendo del movimiento de las partes, si bien no tendrá, como el otro, la suerte de poder reducir este movimiento, simplemente, a tres leyes y dos componentes» (p. 221). 
autores como Jonas o Varela han propuesto que una auténtica teoría de la vida requiere modelos no newtonianos, autoorganizativos, basados en la autonomía o en el funcionamiento del metabolismo ${ }^{3}$.

Recientemente, ciertas controversias internas a la tradición darwinista vuelven a persuadir de la necesidad de considerar los procesos de desarrollo. Así, la crítica al adaptacionismo (Gould y Lewontin 1979) cuestionó la validez epistemológica de las explicaciones que en biología remiten cualquier problema a la selección natural, sin tener en cuenta otros factores como la arquitectura o el Bauplan de los organismos. Las discusiones en torno a la definición de función biológica (Ariew et al. 2002, Wouters 2005) reflejan una insatisfacción similar: mientras que la noción etiológica de función (considerada por muchos aceptada por consenso) defiende que la función de un rasgo es equivalente a la utilidad por la cual fue seleccionada, desde un enfoque kantiano, se ha argumentado que las funciones de las partes de un organismo no pueden determinarse de antemano, dado que se adquieren en el contexto del funcionamiento del todo. La biología evolutiva del desarrollo (conocida por la abreviatura de «evo-devo»), al considerar que el estudio de la evolución requiere ahondar en el conocimiento de los procesos ontogenéticos, ha estimulado el retorno a la comprensión kantiana de los organismos como fines naturales, al menos en cuanto se apela a una noción de organización interna que limita, al tiempo que posibilita, su evolución.

Como vemos, y aunque resulte paradójico, tanto quienes alaban la teoría darwinista de la evolución como quienes la cuestionan reclaman a Kant como predecesor (Zammito 2006). ¿Cómo es esto posible? ¿Cuál de las dos posturas puede calificarse como auténticamente kantiana? ¿La de quienes consideran que Darwin fue el Newton de la hoja de hierba o la de quienes lo niegan? Responder a estas cuestiones requiere un ejercicio de "presentismo histórico" que permita examinar este retorno a la discusión kantiana a la luz de los problemas de la filosofía de la biología actual.

3 «Charles Darwin has often been claimed to be this new Newton of the grass blade, and thus to invalidate Kant's famous dictum. In a way this is not false: the theory of evolution is Newtonian in its character and does attempt to account for design. But, in the end, it seems that Kant had been right in denying a Newtonian, mechanical character of the living: Evolutionary thinking had to re-discover the autonomy and self-organization of the organism and their importance for evolution, so that the Newtonian dominance consequently could be trimmed down considerably, making place for the organism's creativity». (Weber y Varela, 2002, pp. 108-9). 
En este trabajo discutiremos la pertinencia de volver a Kant para fundamentar un proyecto naturalista en el que los problemas filosóficos se planteen a la luz de una teoría científica. Nos ocupamos principalmente de dos cuestiones cuya naturalización ha resultado especialmente problemática: la teleología (y la solución darwinista de entender las funciones biológicas como efectos seleccionados), y el orden taxonómico (que el darwinismo resuelve reinterpretando las relaciones morfológicas a la luz del árbol de la vida).

Ambos problemas han sido afrontados por las distintas tradiciones herederas de Kant en sentidos muy dispares que trataremos de esclarecer articulando nuestro artículo en torno a dos cuestiones. En primer lugar, nos preguntamos hasta qué punto Kant habría considerado a Darwin el Newton de la biología si hubiera conocido su obra. Para ello, analizamos la idea kantiana de ciencia y su epistemología de la naturaleza orgánica (sección 2) y el proyecto darwiniano a la luz de la «Crítica del juicio teleológico» (sección 3), concluyendo que hay al menos un sentido en el que es plausible pensar que Kant se habría sentido satisfecho con Darwin. A continuación, nos preguntamos si la física newtoniana sirve como perspectiva naturalizadora para la biología actual. Dado que nuestra respuesta es negativa, tratamos de justificarla mostrando cómo la evolución de la propia biología requiere un proyecto naturalizador más complejo que el que Newton podría ofrecer (sección 4). En esta línea, dedicamos la última sección a explorar algunas propuestas naturalizadoras que, inspiradas en los desarrollos de las ciencias de la autoorganización y la biología evolutiva del desarrollo, permiten volver a concebir a los organismos como «fines naturales».

\section{El problema de la teleología en la crítica kantiana}

\subsection{Los organismos como fines naturales}

En la Crítica de la Razón Pura, Kant asentaba las condiciones de la investigación científica mediante una distinción radical entre Entendimiento y Razón: el Entendimiento se ocupa de los fenómenos, mientras que la Razón es una facultad productora de cierto tipo de representaciones (ideas) que apuntan a lo inteligible (noúmenos). Así, el conocimiento científico (Naturwissenschaft) es obra del Entendimiento, que es capaz de establecer juicios sintéticos a priori, resultado de la síntesis entre los conceptos puros de la razón y las intuiciones 
sensibles ${ }^{4}$. Como es bien sabido, este tipo de juicios fue forjado por Kant a imagen y semejanza de los conceptos y las leyes universales de la física newtoniana. En ellos, la causalidad se concibe como fruto de la sucesión de los fenómenos en el espacio y el tiempo, donde sólo lo anterior condiciona lo posterior y la interacción entre las partes determina el todo.

Obviamente, existe una región de la naturaleza que parece desafiar este esquema: los organismos — reconoce Kant - aparentan estar regidos por un tipo de causalidad (a saber, la causalidad teleológica) que contradice el tipo de causalidad mecánica 5 .

Resolver esta aparente contradicción es el objetivo de la segunda parte de la Crítica del Juicio, dedicada al juicio teleológico. En ella, Kant se enfrenta al problema de la naturaleza teleológica de los seres naturales organizados (Cfr. Ginsborg 2008), que analiza en contraposición con la de los artefactos:

Así como en un producto semejante de la naturaleza, cada parte existe sólo mediante las demás, de igual modo es pensada como existente sólo en consideración de las demás y del todo, es decir, como instrumento (órgano). Pero eso no basta [...] sino que ha de ser pensada además como un órgano productor de las otras partes (por consiguiente, cada una a su vez de las demás), tal como no puede serlo ningún instrumento del arte, sino sólo de la naturaleza, la cual pro-

${ }^{4}$ Kant define la facultad de Juicio como «la capacidad de concebir lo especial como contenido dentro de lo general» (Kant, CJ, Introducción, p. IV), pues el Juicio, si no quiere disolverse en el fragmentario y caótico magma fenoménico, aspira por definición a «un conocimiento conexo de la experiencia según una general conformidad de la naturaleza a leyes» $(C J, \S 70)$. Sin embargo, el ordenamiento de los objetos empíricos en conceptos y su entrelazamiento en leyes no siempre es necesario. Lo será cuando un principio objetivo le sea dado al Juicio por medio del entendimiento. En tal caso, el Juicio es determinante, porque la Razón se funda en leyes universales que el entendimiento prescribe a priori a la Naturaleza.

5 Existe un debate sobre el modo en el que Kant entiende la causalidad o el mecanismo en la Crítica del Juicio. Podemos distinguir tres posturas (Ginsborg 2008): 1) algunos comentadores consideran que se trata de una noción de causalidad equivalente a la establecida por Kant en su Crítica de la Razón Pura. Desde esta perspectiva, el principio de mecanismo sería regulativo, lo que no tiene sentido en la CJ; 2) McLaughlin (1989, 1990) defiende que el concepto de mecanismo en la CJ responde a una causalidad específica: la determinación del todo por sus partes; 3) Ginsborg (2001) defiende que algo puede ser explicado «mecánicamente» si puede explicarse su existencia en virtud de los poderes intrínsecos de la materia a partir de la cual se conforma. 
porciona toda materia para instrumentos [...], y sólo entonces y por eso puede semejante producto, como ser organizado y organizándose a si mismo, ser llamado un fin de la naturaleza. (Kant, $C J$, $\$ 65$ ).

Como vemos, el concepto kantiano de «fin de la naturaleza» (Naturzweck) no hace referencia ni a la intención ni a la utilidad, sino a la relación entre todo y parte, paralela a la distinción entre medios y fines, que se produce en el interior de los organismos: un organismo es un fin natural en el sentido de que es «tanto la causa como el producto de sí mismo» (\$ 64). Para ello, es decir, para que un objeto sea un fin natural, deben darse dos condiciones $(\$ 65)$ : en primer lugar, las partes orgánicas deben ser fines en el sentido de que sólo sean posibles a través de su relación con el todo, es decir, las partes de un fin natural tienen funciones. Esta es la definición de fin y, por tanto, se aplica también a artefactos como los relojes, donde las partes sólo pueden comprenderse en virtud del rol que cumplen en el todo. La segunda condición es exclusiva de los fines naturales: a diferencia de lo que sucede con las de un reloj, las partes de un organismo son, recíprocamente, causa y efecto de su forma. Los organismos son totalidades autoproductoras que no requieren de ningún agente externo, pues sus partes se producen recíprocamente. Nos encontramos, en este caso, ante una idea de función que no puede determinarse de antemano.

La singularidad teleológica de los organismos se revela en las propiedades vitales que manifiestan: 1) la autorreproducción, por la que un organismo se replica «en especie»; 2) la autoproducción, por la cual el organismo se construye a sí mismo (el desarrollo es en sí mismo un concepto de fin, pues presupone un «sujeto» unitario de los fenómenos vitales que, transformándose, se mantiene igual a sí mismo a través de todos los cambios, actuando como principio orientador y no como resultado); 3) el automantenimiento, por el que los organismos perseveran como individuos mediante tres vías principales: autorreparándose, tomando alimento de fuera y convirtiéndolo en el tipo de sustancia orgánica de la que él mismo está compuesto, y manteniendo su estructura fisiológica mediante la dependencia recíproca de sus partes.

Como vemos, el concepto de vida implica necesariamente para Kant un tipo de acción que no va de las partes al todo, sino del todo a las partes. Por tanto, aunque, al igual que todos los objetos de nuestra experiencia, los organismos están sometidos al principio mecánico, su carácter irreductiblemente holista hace 
que las leyes causales que aplicamos a los objetos físicos resulten inapropiadas para comprenderlos.

El fenómeno de los seres naturales organizados parece, por tanto, obligarnos a concebirlos bajo el concepto de fin. Pero si este fuera el caso — dice Kant— los objetos orgánicos estarían constituidos por principios contradictorios: por un lado, obedecerían a leyes mecánicas, pues la subsunción de los fenómenos en leyes universales es la condición de posibilidad de toda experiencia; por otro, estarían gobernados por las leyes particulares de la teleología, a la que la causalidad mecánica habría de estar subordinada. Pero esto, según la Crítica kantiana, es imposible: sólo accedemos a los fines naturales a través de la experiencia, y nuestra razón no puede determinar a priori que el fenómeno que va a ser percibido responda a la causalidad final.

Lo que en realidad sucede cuando juzgamos a los organismos como fines naturales — argumenta Kant- es que nuestro Juicio, ante la disparidad de las leyes particulares, se ve obligado a fundamentarse en sí mismo para encontrar un hilo conductor que organice la multiplicidad natural. La Razón, entonces, se representa la idea de un todo y la proyecta sobre el producto natural organizado que trata de comprender. Pero, en este caso, lo que está actuando como causa no es la propia totalidad, sino su imagen. El juicio no es aquí un juicio determinante sino reflexionante; no determina a los objetos, sino que reflexiona sobre ellos. La teleología no es, por tanto, un principio objetivo de la naturaleza (como lo es la causalidad mecánica), sino un principio subjetivo de nuestra Razón (\$ 75).

El planteamiento crítico del problema de la teleología no implica negar que, en el fondo por nosotros desconocido de la naturaleza, las causalidades mecánica y final estén, en los organismos, conectadas en un sólo principio, sino que su objetividad es indemostrable para nuestro entendimiento (\$ 70). La teleología - concluye Kant- pertenece al terreno de lo nouménico, y sólo un entendimiento intuitivo, propio de una «inteligencia arquetípica», podría concebirla como principio objetivo de la naturaleza. Así, mientras que nuestro entendimiento sólo nos permite pensar en un todo como efecto de las fuerzas motrices de sus partes, podemos pensar en «una facultad de conocer distinta de la sensibilidad y totalmente independiente de ella», un entendimiento intuitivo que, en lugar de ir de las partes al todo, iría de lo sintético-universal (de la intuición de un todo) a lo particular, es decir, del todo a las partes ( $\$ 77)$. 
Ahora bien: como de lo suprasensible no podemos tener más que el concepto indeterminado de un fundamento que posibilita el Juicio de la naturaleza según leyes empíricas, es decir, como se trata de un principio trascendente, entonces no podemos hacer descansar en él la explicación de la posibilidad de los organismos según leyes dadas para el Juicio determinante, sino sólo la exposición de la misma para el reflexionante.

De este modo, la investigación de los seres naturales organizados puede estar regida por dos principios no contradictorios: según la máxima del entendimiento, todo organismo debe ser juzgado según causas mecánicas; según la máxima del juicio reflexionante, los seres organizados han de juzgarse según causas finales, «sin querer, por eso, decidir por este principio nada sobre la posibilidad misma de semejantes cosas» (\$78). Así, queda justificada la necesidad de que el Juicio reflexionante, en su legítima búsqueda de la regularidad natural, trame en la propia praxis de la observación empírica el «hilo conductor» que, orientado por la finalidad, entreteja las formas en las que se nos presentan los seres organiza$\operatorname{dos}(\$ 79)$.

Como vemos, el carácter subjetivo del principio teleológico no impide a Kant reconocerle una función radical en la investigación científica; una función regulativa que no se opone a la explicación causal, «sino que la prepara, señalándole los fenómenos y los problemas sobre los que ha de proyectarse», generando las unidades que en nuestro proceder analítico segmentaremos en sus distintos elementos causales. Así, la investigación crítica, que parecía anunciar una defunción definitiva del principio teleológico, acaba resucitándolo de entre los escombros: desde una perspectiva metafísica, el concepto de fin es, en efecto, aquel asylum ignorantiae del que hablaba Spinoza, pero metodológicamente se convierte en el medio para un conocimiento cada vez más exhaustivo de los engarces y las relaciones estructurales de la naturaleza orgánica (Cassirer 1974, pp. 400-402).

\subsection{Hacia una arqueología de la naturaleza}

La particular relación entre todo y parte aparece también cuando consideramos no ya cada producto natural organizado, sino la totalidad de los organismos. Al describir la multiplicidad de las formas orgánicas, ordenamos en siste- 
mas las semejanzas y diferencias que percibimos entre ellas, agrupándolas en categorías taxonómicas jerárquicamente anidadas. Este orden que aparentemente percibimos en las formas naturales puede conducirnos a suponer algún tipo de unidad morfológica que subyazca a esas múltiples partes que se resolverían como manifestaciones suyas.

¿Cómo se aplica el análisis crítico de la teleología al problema del orden taxonómico? ¿Cómo se relacionan aquí el juicio determinante y el reflexionante? En principio, las afinidades orgánicas se nos presentan como una realidad «dada», lo que nos permite clasificar las formas orgánicas en diferentes categorías taxonómicas. Sin embargo — dice Kant—, la descripción de la naturaleza sólo puede valerse de la imaginación. La idea de que el material biológico puede ser ordenado y clasificado en "géneros» y «especies» no es más que un postulado que nuestra experiencia de ningún modo está obligada a acatar.

Lo que ha de hacer el naturalista, si quiere convertir su labor en un proyecto científico, es indagar las causas de esta aparente identidad morfológica, y la elucidación de los mecanismos que explican el orden taxonómico no pertenece ya a una descripción sino a una teoría de la naturaleza. Para ello, la variedad de las morfologías orgánicas no puede considerarse una variedad dada, pues entonces estaríamos suponiendo una unidad subyacente que nuestro entendimiento no puede aprehender. Sin embargo, si consideramos que la variedad de las formas naturales no es una variedad dada sino creada, es decir, si concebimos las formas orgánicas como el eslabón actual de la sucesión de otras tantas formas en el tiempo, sí podemos someter esta secuencia al mecanismo causal que autoriza la configuración de nuestro entendimiento. En este sentido, podemos aplicar el juicio determinante, estableciendo la secuencia temporal en la que se han sucedido las formas orgánicas. La «afinidad formal» se convertirá, así, en una afinidad genealógica:

Esa analogía de las formas, en cuanto, a pesar de toda la diversidad, parecen ser producidas según un prototipo común, fortalece la sospecha de una verdadera afinidad de las mismas en la producción de una madre común primitiva, por medio de la aproximación gradual de una especie animal a otra. $(\$ 80)$.

La anatomía comparada actúa aquí como principio regulativo, orientándonos en la búsqueda de «algo semejante a un sistema según el principio de producción» (\$ 80). Si recorremos el camino inverso al del relato y, en lugar de arran- 
car de la Creación, partimos de las «hechuras actuales de las cosas», entonces será posible acatar la máxima establecida por la Crítica del juicio, según la cual,

Es [...] razonable, y hasta meritorio, seguir el mecanismo de la naturaleza para una explicación de los productos naturales, tan lejos como ello pueda hacerse con verosimilitud y no abandonar este ensayo porque sea imposible en sí coincidir por su camino con la finalidad de la naturaleza, sino sólo porque ello es imposible para nosotros hombres, pues se exigiría para ello una intuición diferente de la sensible y un determinado conocimiento del substrato inteligible de la naturaleza que pudiera dar fundamento también al mecanismo de los fenómenos según leyes particulares, todo lo cual supera totalmente nuestra facultad $(\$ 80)$.

De este modo, a diferencia de la descripción, la historia de la naturaleza queda remitida al entendimiento, facultad que, más allá de reconocer semejanzas, establecería las leyes que fundamentan los lazos de parentesco, es decir, las leyes de la conexión reproductiva ${ }^{6}$ (Kant 2004, pp. 36-37).

La historia de la naturaleza legitimada por Kant en su ensayo «Sobre el uso de los principios teleológicos en la filosofía» contiene, de hecho, los dos postulados fundamentales que determinarán la elección darwinista del mecanismo rector de lo que a partir de finales del XIX empezará a llamarse «evolución». En primer lugar, para el desvelamiento de la causalidad mecánica rectora de la evolución orgánica, Kant exige partir de la analogía con fuerzas conocidas que actúen en el presente. Se adelanta así al postulado que dirigirá el actualismo geológico de Lyell y que Darwin retomará al partir de la selección artificial como análogo de la selección natural. En segundo lugar, Kant impone un límite definitivo a la exploración mecánica de la evolución biológica: el origen de la vida; un límite radical, porque impide que podamos alcanzar, a través del mecanicismo, una solución definitiva al problema que depara la aparente afinidad de las formas naturales.

${ }^{6}$ Alvarez (1998) considera la distinción kantiana entre las especies naturales (que conducen a una clasificación natural, basada en la capacidad reproductiva) y las académicas o artificiales, subrayando que «[1] a crítica de Kant al Systema Naturae de Linneo se basaba, precisamente, en que sus grupos básicos no son naturales, sino especies académicas, artificiales, por lo cual dicho sistema no pasa de ser una clasificación lógica» (pp. 57-58). De un modo similar, Sloan (2001) relaciona la descripción de la naturaleza con el trabajo de Bacon y Linneo, y la historia de la naturaleza con el de Buffon, Woodward y Robinson. 
A pesar de la solución que nos ofrece la contemplación de la diversidad orgánica como una variedad creada, Kant advierte que la historia de la naturaleza no podrá aspirar jamás a conquistar el estatuto definitivo de cientificidad. Pues, por mucho que el historiador de la naturaleza someta la evolución a la investigación genética del mecanismo causal, acabará por toparse con la cuestión del origen. Para poder construir la historia de las especies resulta, por tanto, imprescindible que se ponga «siempre a la base alguna organización primitiva que utilice aquel mecanismo para producir otras formas organizadas o desarrollar la suya en nuevas figuras» $(\$ 80)$. De modo que, por mucho que avanzase el análisis causal mostrando de un modo puramente mecánico cómo el siguiente eslabón de la cadena evolutiva se deriva y nace del anterior, por mucho que nos remontásemos, acabaríamos topándonos siempre con un estado inicial de «organización». Y puesto que el concepto de organización no puede comprenderse mecánicamente sino que exige una explicación teleológica, «no puede indagarse en la física de dónde proviene originariamente toda organización». El enemigo dialéctico es aquí la teoría de la generación espontánea, una forma de «epigenetismo radical», consecuencia de aquellas hipótesis, como las de Herder o Blumenbach, que habían concebido las razas y las variedades como producto de circunstancias ambientales contingentes (Huneman 2006). Para Kant, la consideración causal puede enseñarnos con sujeción a qué reglas se pasa de una estructura a otra, pero lo que no puede hacernos comprender es la existencia de los «embriones» individuales que sirven de punto de partida de la evolución (Cassirer 1974, pp. 402-403). La existencia de una organización originaria ha de concederse como premisa, pues la explicación de su origen pertenecería no al ámbito de la ciencia sino al de la metafísica (Kant 2004, p. 26). Sin embargo, si hacemos epojé de este origen, podemos aplicar el principio del mecanismo a la analogía de las formas orgánicas, de manera que la evolución logre explicarse de un modo puramente causal en todas y cada una de sus fases. Como veremos más adelante, este será el «triunfo de Darwin».

\subsection{La doble herencia de Kant}

La poderosa influencia ejercida por la filosofía kantiana se bifurca durante el Romanticismo en dos direcciones (Albarracín 1983, p. 39): por un lado, el idealismo especulativo post-kantiano de Fichte, Schelling y Hegel; por otro, la filosofía neokantiana de Fries y Apelt, y de Herschel y Whewell en el mundo anglo- 
sajón. Ambas trayectorias encuentran su traducción más preclara en biología, donde la distinción kantiana entre variedad dada y creada contiene las dos grandes líneas en las que se bifurcarán las interpretaciones de la unidad y la diversidad morfológica: la morfología trascendental y la teoría darwinista de la evolución?

La morfología transcendental se inspira en la obra de Kant, a la vez que se rebela contra los límites que éste impone al conocimiento. La anatomía filosófica francesa y, de un modo más explícito, la filosofía natural alemana, heredan la concepción de la teleología de la Crítica del Juicio. Como vimos, en la obra kantiana la causalidad final se distingue de todas las demás categorías en que, mediante ella, se afirma un nuevo tipo de «unidad de lo múltiple» en la que el todo no es un conglomerado de partes, sino su fundamento originario. La morfología trascendental se propone investigar la que Kant había considerado la característica esencial del reino orgánico: la subordinación de las partes al todo que se produce tanto en cada organismo particular como en la totalidad de las formas biológicas, consideradas como partes o manifestaciones de un tipo o totalidad subyacente. Sin embargo, en lugar de negar a la razón humana la capacidad de aprehender la teleología, como hiciera Kant, la morfología racional la afirma. Con el fin de recuperar la cognoscibilidad del noúmeno y, por tanto, del organismo como una entidad objetivamente teleológica, la filosofía natural romántica apostó por «humanizar» la intuición, de modo que sujeto y objeto pudieran volver a fundirse en una sola sustancia. De este modo, el noúmeno deja de ser un sustrato oculto más allá de los fenómenos, manifestándose en ellos mismos. Mientras que en la investigación crítica la «sujeción formal a un fin» no puede ser más que un principio regulativo, en la filosofía natural, el «tipo», en tanto que dimensión interna de la multiplicidad fenoménica, puede ser aprehendido por la intuición (Bortoft, en Goethe 2002, p. 17). Así, para la morfología trascendental, la variedad biológica vuelve a ser una variedad dada, en tanto que manifestación de un tipo subyacente. Ese fue el gran proyecto de investigación de la morfología idealista: orientar la anatomía comparada a la búsqueda de los «planes de organización» que fundamentaban la multiplicidad orgánica.

7 Richards (2002, p. 229. Cit. en Zammito 2006) sostiene que, en realidad, aquellos filósofos o naturalistas decimonónicos que encontraron alguna afinidad en su proyecto fue, o bien por incomprensión (Blumenbach y Goethe) o bien por la reconstrucción de ciertas ideas con consecuencias muy distintas a las pretendidas por Kant (Kielmeyer y Schelling). En realidad, tanto el darwinismo como la morfología trascendental reconstruyeron a Kant. 
Sin embargo, como hemos visto, la vía de investigación auténticamente legitimada por la "Crítica del juicio teleológico» es aquella que indague la afinidad formal entre los seres orgánicos en tanto que producto generado en una evolución histórica. Kant había considerado su especulación evolucionista como «una audaz aventura de la razón», frustrada, en gran parte, por la ausencia de datos paleontológicos que permitieran reconstruir con rigor científico una historia semejante. Como enseguida comprobaremos, el desarrollo de la geología y la paleontología en las décadas previas a la publicación del Origen ofrecerá suficiente material empírico como para que mereciese la pena correr un riesgo semejante.

\section{Darwin y la ciencia de los organismos}

Como apuntábamos en la introducción, llamar a Darwin «el Newton de la hoja de hierba» no es una analogía meramente retórica. La peculiar concepción darwinista del cambio orgánico y la apuesta por una causalidad eficiente acabarán exorcizando a la forma y la teleología del dominio evolutivo, como Newton hiciera con el movimiento. Sin embargo, el viraje filosófico del darwinismo es muy distinto al de la revolución newtoniana: si en física la revolución mecánica fue la responsable del giro kantiano, en biología es este último, al universalizar las exigencias metodológicas de la física a la totalidad de la ciencia, el que provoca la perturbación filosófica de la investigación biológica.

El Origen de las especies ofreció una doble solución a la secular dialéctica que asolaba a la biología decimonónica (Gilbert 2003): por un lado, la comunidad de descendencia daba cuenta de la "unidad de tipo», de modo que las semejanzas morfológicas lograban ser explicadas genealógicamente a través de un origen común; por otro, la teoría de la selección natural explicaba la diversidad orgánica en virtud de las adaptaciones particulares de los organismos a sus «condiciones de existencia». Como trataremos de demostrar a continuación, esta doble revolución darwinista cumplía con las credenciales epistemológicas que Kant había exigido al naturalista para enfrentarse científicamente al problema de la diversidad taxonómica.

Desde la aparición de los sistemas de clasificación en la primera mitad del siglo XVIII, la tensión entre descripción y explicación era cada vez más insostenible en taxonomía. Es en este contexto en el que Lamarck acuña en 1802 el tér- 
mino «biología»: si la historia natural se limita a la recopilación exhaustiva de hechos y datos, la biología habrá de perseguir las leyes fundamentales de la vida. Como vemos, se trata de una distinción paralela a la distinción kantiana entre la descripción y la historia de la naturaleza. Para Lamarck, este ejercicio teórico requería una filosofía de la naturaleza $(1873$, pp. 69-70) fundamentada en el fisicalismo y el mecanicismo ejemplificados por la ciencia newtoniana. Sin embargo, el Origen no aspira ya a fundamentarse en una filosofía de la naturaleza, sino en la filosofía de la ciencia impuesta por el espíritu neokantiano, preocupada por la estructura que hace científica a una teoría, el modo en el que ha de estudiarse la naturaleza al margen de sus esencias.

El marco epistemológico en el que se inserta la revolución darwinista está reconocidamente dominado por la filosofía neokantiana de John Herschel y William Whewell ${ }^{8}$. La irrupción del espíritu crítico en el terreno de la clasificación biológica, atravesado por el nominalismo y el funcionalismo anglosajón, da lugar a dos revoluciones teóricas con consecuencias fundamentales para el estatuto de la teleología en la biología evolucionista. En primer lugar, la distinción kantiana entre la descripción y la historia de la naturaleza se traduce en la diferencia entre taxonomía y sistemática filogenética; en segundo lugar, la causalidad rectora del nuevo orden histórico se reduce a causalidad eficiente, logrando así la externalización del cambio en el reino orgánico: al igual que el movimiento, la transformación biológica deja de atribuirse a una forma inmanente para convertirse en resultado de una fuerza externa: la selección natural.

\subsection{El árbol de la vida: de la taxonomía a la sistemática filogenética}

$\mathrm{Al}$ igual que Kant, Darwin denuncia la taxonomía como mera recopilación de hechos y aboga por una teoría de la naturaleza en la que las clasificaciones

${ }^{8}$ Darwin no se compromete explícitamente con ninguna filosofía de la ciencia, pero el marco epistemológico de Herschel y Whewell se demuestra en el ejercicio y es declaradamente reconocido en otros documentos. A pesar de la adversa reacción de ambos ante la teoría de la evolución, el modelo de ciencia legitimado por ambos, fundamentado en el método hipotético-deductivo y la noción de vera causa, fue un referente esencial para la articulación de la teoría de Darwin (Cfr. Ruse 1975). 
reflejen el «sistema natural» (Origen, p. 552). El criterio de «naturalidad» de los sistemas taxonómicos es también auténticamente kantiano, pues Darwin identifica la legalidad con el orden en el tiempo, definiendo las leyes naturales como «la sucesión de hechos» (Origen, p. 138). Aplicado a la historia natural, esto significa, en términos modernos, que la taxonomía se convierta en sistemática filogenética: la clasificación habrá de ser un sistema natural que refleje el orden seguido por la naturaleza para generar las formas que hoy observamos. De este modo, Darwin aplica a la evolución el concepto de ley natural heredado de la física y legitimado por la Crítica kantiana: la biología investigará la variedad orgánica en tanto que resultado de una evolución histórica, entendiendo por ésta un proceso mecánico en el que la causalidad no es ya resultado de la disposición espacial de las partes, sino del encadenamiento de sucesos en el tiempo (González Recio 2004, p. 231). La postulación darwinista del «principio genealógico o de descendencia» como «el oculto lazo de unión que los naturalistas han buscado con el nombre de sistema natural» (Origen, p. 576) tendrá dos consecuencias fundamentales para la taxonomía (Insua Rodríguez 2005): por un lado, el principio de continuidad que había caracterizado a la idea de scala naturae deja de ser metafísico para traducirse en la continuidad histórica entre ancestro y descendiente (Gould 2004, p. 175); por otro, las relaciones genealógicas entre los taxones explican la estructura jerárquica de la sistemática linneana: el género hace a los caracteres, y no a la inversa, precisamente porque las especies que lo conforman descienden de un antepasado común del que han heredado sus caracteres "genéricos».

Ahora bien, ¿qué sucede con el origen del árbol de la vida? Como vimos arriba, la Crítica del Juicio había legitimado la edificación de una taxonomía histórica siempre que el hilo temporal que enlazara las transformaciones orgánicas se detuviese en alguna forma primera que fundaría el origen de la secuencia y que sólo podía ser comprendida por un entendimiento divino. A diferencia de Lamarck, cuyo recurso a la generación espontánea implica un compromiso materialista (el movimiento de la materia provocado por fuerzas naturales es capaz de generar los organismos más sencillos) (Bowler 1995, p. 157), Darwin acata la prohibición kantiana: la investigación biológica no podrá adentrarse nunca en lo que Herschel calificó como «el enigma de los enigmas» (Ruse 1983). El mecanismo causal de la evolución no podía interferir, por tanto, con la cuestión del origen. Como en Kant, «el origen» no hace referencia aquí al origen de cada especie, sino al de aquella primera forma a partir de la cual habrían de derivarse todas las demás. De hecho, Darwin nunca llegó a comprometerse con el monofiletis- 
mo, sino que postuló «varios poderes [los «principios de producción» de los que hablaba Kant] que originalmente habrían sido insuflados en unas pocas formas o en una» (Origen, p. 490). A partir de la existencia de un número reducido de formas orgánicas sobre cuya génesis no se posiciona, la legalidad natural (la selección natural, en este caso) puede empezar a funcionar. Por eso Darwin no admite la no explicación del origen como objeción a su teoría. Muy al contrario, tal y como había recomendado Kant, la epojé del origen actuaba como una premisa necesaria del «largo argumento» de El Origen de las especies.

\subsection{El mecanismo del cambio: la selección natural}

Mientras que la concepción continental de la dinámica orgánica (inspirada en el desarrollo) se fundó en un sujeto invariante en el cambio, la revolución darwinista exigía localizar un mecanismo transformador externo a los seres sujetos a él, una vera causa mecánica que explicase la transformación biológica, exigencia común a las filosofías neokantianas de Herschel y Whewell. De acuerdo con Herschel, una explicación fundamentada en la vera causa sólo invoca causas (preferiblemente fuerzas) que pueden ser observadas directamente, independientemente del efecto que quiere explicarse. Para Darwin, su teoría se ajustaba perfectamente a estos preceptos: la variación y la lucha por la vida eran directamente observables en una población, al margen de su influencia en el cambio evolutivo.

El trabajo de Malthus resultó fundamental para concebir el cambio evolutivo en términos mecánicos. El crecimiento geométrico se convirtió en una ley fundamental de la dinámica poblacional, desempeñando un papel similar al de la primera ley de Newton en física (Ginzburg y Colyvan 2004). Así, el crecimiento poblacional puede concebirse como una «ley ecológica de la inercia» que describe el comportamiento del sistema en ausencia de perturbaciones: el estado predefinido de una población no es el reposo (una población constante), sino el movimiento (el crecimiento o declive exponencial), de modo que cuando las poblaciones no crecen o disminuyen exponencialmente, sabemos que una fuerza externa (ambiental) está alterando las tasas de natalidad y/o mortalidad.

La concepción de la evolución según los esquemas epistemológicos de la dinámica requería importar dos aspectos fundamentales de la mecánica clásica. En 
primer lugar, al igual que la física newtoniana se fundamentó en un análisis del continuo que permitió el seguimiento de las relaciones causales entre los fenómenos, las dinámicas geológica y biológica, si querían ser investigadas científicamente, debían regirse por leyes que actuasen en un tiempo homogéneo y uniforme: si se admitía el gradualismo geológico de Lyell y se consideraba que los cambios individuales eran una respuesta a cambios ambientales, el cambio específico también debía ser muy lento (González Recio 2004). En segundo lugar, la consideración de la evolución como resultado del efecto acumulado de pequeños cambios sucesivos requería la continua actuación de las mismas leyes físicas (actualismo): como la fuerza gravitatoria sobre la totalidad de los cuerpos, la presión selectiva actúa constantemente sobre los organismos, independientemente de sus esencias. El cambio, como el movimiento, se externaliza de los seres sujetos a él: los organismos no imponen restricciones a la selección natural y, por lo tanto, no intervienen en la dirección de la evolución. Aquí encontramos la diferencia radical entre el transformismo darwinista y el resto de mecanismos evolutivos propuestos hasta entonces: mientras que en aquellos la teleología interna de los organismos jugaba un papel primordial, en la teoría de la selección natural toda causalidad formal-final interna desaparece.

Pero si quería mantenerse el carácter externo de la fuerza transformadora, la variación modelada por la selección natural había de ser aleatoria, isotrópica y gradual, pues si los cambios evolutivos pueden ser producto de variaciones discontinuas, la creatividad residiría en la variación misma (Gould 2004, p. 171). Darwin no cree que la variación individual sea casual, pero ya no apela a la causalidad formal-final. Las variaciones — reclama el Origen - han de obedecer necesariamente a una causa eficiente (pp. 31, 263, 563). Son muchas, de hecho, las causas que Darwin admite como generadoras eficientes de variación, pero la causalidad última que dirige el proceso evolutivo se localiza en la selección natural, una fuerza que actúa sobre las variaciones independientemente de su origen. La causalidad interna deja de ser, por tanto, explicativa. De ahí que la teoría de la herencia a la que se adhiere Darwin (la pangénesis), aunque falsa, tuviera un efecto menor en la estructura de la teoría de la evolución. La selección natural no requería una teoría sobre la herencia; sólo necesitaba disponer de una cantidad de variabilidad abundante (Mayr 1992, p. 95). Así, de hecho, lo reconoce Darwin cuando afirma que el desconocimiento de la esencia de los mecanismos hereditarios no nos impide estudiar y conocer sus efectos (Ruse 1983, p. 294). 
De este modo, como subraya Walsh (2006), todos los rasgos que, para Kant, permitían identificar a los organismos como «fines naturales» se convierten en meras consecuencias del cambio en la estructura poblacional llevado a cabo por la selección natural, lo que condujo a Mayr a proclamar que «Darwin había resuelto el gran rompecabezas de Kant».

El problema es que, en realidad, los conceptos de forma y función a los que Darwin se enfrentó en el Origen eran muy distintos de los manejados por la tradición continental (Cfr. Etxeberria y Nuño de la Rosa 2009b). Como vimos arriba, la adaptación funcional de los naturalistas continentales es una función inmanente a los organismos, fundada en el principio de coordinación de las partes. Sin embargo, el objetivo del Origen no es explicar el problema de la teleología, tal y como había sido planteado por Kant, sino dar cuenta de «la perfecta adaptación de las especies a su entorno» (p. 3). Y es que Darwin no parte de los interrogantes que la morfología había heredado de la Crítica kantiana, sino del argumento del diseño de la teología natural británica y de las cuestiones que asolaban a la sistemática y la historia natural, a saber: el problema de las especies y de la adaptación ecológica (Russell 1916). En el darwinismo, la función biológica ya no es un modo de ser, sino una forma de ajustarse al entorno. Podríamos decir que Darwin hereda la noción kantiana de ciencia, pero no la problemática que ésta trata de resolver: el Origen aplica la solución crítica que convierte a la descripción en una historia de la naturaleza regida por un mecanismo eficiente (la selección natural); pero la aplica a un problema que no es el de la subordinación teleológica de las partes al todo orgánico, sino el de la adaptación, un concepto de función que Kant había considerado secundario y subordinado a la teleología intrínseca.

\section{Darwinismo y teleología}

\subsection{El papel de la noción de organismo en la biología evolutiva}

El tránsito del darwinismo newtoniano al estadístico que inauguró la genética de poblaciones estuvo marcado por la "desaparición» del organismo como entidad fundamental de las explicaciones biológicas.

Dado que en genética de poblaciones la variabilidad del sistema genético se considera ilimitada, la evolución puede interpretarse como una exploración alea- 
toria del espacio de secuencias genéticas, en el que la selección asume el papel de «filtro» funcional externo (Garcia-Azkonobieta 2005). La teoría evolutiva se convierte, así, en una pura «cinemática formal» que abstrae las cualidades de los objetos que evolucionan (Gayon 1998), de forma que los organismos, centrales en el trabajo original de Darwin, dejan de cumplir un papel relevante (Gould 2004).

Este giro epistemológico se manifiesta del modo más evidente en la obra de Dawkins (1976), para quien la vida comienza con la emergencia de un replicador y los organismos son meramente máquinas de supervivencia construidas por estos. De hecho, esta reinterpretación del darwinismo orienta su investigación al estudio de cómo entidades supra-orgánicas (las poblaciones) cambian como resultado de los poderes causales de entidades sub-orgánicas. En ese proceso de «endurecimiento de la Síntesis Moderna» (Gould 1983), los replicadores se convierten en las únicas entidades que participan en todos los procesos constitutivos de la evolución: la herencia (pues sólo se heredan los replicadores), la ontogenia (que se concibe como programada por estos) y los cambios poblacionales (dado que sólo son evolutivos aquellos cambios en la frecuencia de los replicadores). Esta perspectiva reduccionista permite que las partes (los replicadores) puedan explicar el todo (los organismos) sin ninguna necesidad de apelar a la teleología (Walsh 2006). Por esta razón, podemos decir que la integración en biología evolutiva de la consideración de cómo son los organismos que evolucionan (su morfología, su ontogenia, etc.) permite recuperar una concepción de los seres vivos, en la estela de Kant, como entidades organizadas mucho más complejas que los artefactos.

\subsection{El árbol de la vida}

En lo que afecta al árbol de la vida, las discusiones sobre si es la morfología o la mera genealogía lo que va a permitir construir clasificaciones más naturales de la diversidad orgánica han sido constantes en la biología evolutiva, sobre todo desde que a partir de los años cincuenta del siglo XX una metodología genealógica radical, la cladística, emergiera con enorme vigor, desplazando a la escuela fenética, partidaria de una taxonomía numérica basada en similitudes y diferencias.

Es en el marco de esta discusión cuando el efecto de la distinción kantiana entre la descripción y la historia de la naturaleza se reconoce de un modo más explícito. Patrick Tort y David Hull distinguen entre la clasificación fundada en 
la genealogía (la taxonomía evolucionista y la cladística) y la asentada en la semejanza (Cfr. Blanchette 2002). La primera —argumentan — utiliza la memoria y tiene por objetivo ordenar a los seres orgánicos bajo rúbricas, mientras que la segunda hace uso del entendimiento, que trata de hacerlo según leyes. La estrategia basada en la semejanza se admite como primer paso de la investigación, e incluso como la más intuitiva, pero su anterioridad se declara exclusivamente epistemológica, o regulativa, en terminología kantiana: las verdaderas clasificaciones naturales se fundamentan en relaciones filogenéticas.

Como veremos, la Síntesis moderna y la cladística eliminan el problema de la «unidad en la multiplicidad», estableciendo una completa redefinición de los conceptos de homología y plan corporal: ambos dejan de caracterizarse por su identidad morfológica para hacerse depender, exclusivamente, de su ascendencia común. La genealogía no es ya la causa de la identidad estructural, ya no explica sino define las formas. En este nuevo marco teórico, los criterios anatómico y embriológico dejan de pertenecer a la definición de los taxones y las homologías para convertirse en criterios meramente metodológicos de reconocimiento de la ascendencia común. De este modo, la homología se independiza definitivamente tanto de la función como de la forma y estructura de los órganos (Mayr 1982).

\subsection{La evolución del darwinismo}

Si bien los comentadores del darwinismo (como Gayon 2003) han considerado que en biología nunca se ha dado una revolución que desbanque a Darwin de su posición de «figura pionera» (como sí sucedió en física) y que todos los cambios de perspectiva han conservado la centralidad de la obra darwiniana, no cabe duda de que el darwinismo ha sufrido profundas transformaciones en los ciento cincuenta años que han transcurrido desde la publicación del Origen. Como veremos, si bien Darwin, siguiendo a Kant, había reservado para el organismo cierto rol regulativo, y reconocido en la organización un límite infranqueable al enfrentarse al origen de la vida, el darwinismo ortodoxo ha ido más allá, sobrepasando los límites impuestos por Kant a la investigación de la naturaleza orgánica.

Un trabajo historiográfico pionero en el análisis de la evolución del darwinismo es el de Depew y Weber (1995), donde se analiza la transformación del 
darwinismo en función de la interpretación física del concepto de selección natural en tres épocas o perspectivas diferentes: el darwinismo newtoniano, el darwinismo estadístico y el darwinismo autoorganizativo.

La primera etapa se caracteriza por el modo en que Darwin y sus contemporáneos entendieron la selección natural. Como vimos, el hallazgo de un mecanismo en armonía con los principios de la dinámica newtoniana fue la pieza clave de la originalidad e importancia de la teoría de Darwin en relación al pensamiento evolucionista de su época. Así como una fuerza externa, la de la gravedad, constriñe el comportamiento inercial de los cuerpos en la física newtoniana, para Darwin la trayectoria transgeneracional de los sistemas biológicos, que implicaría un crecimiento demográfico incontrolado, está limitada y moldeada por una selección externa.

Los artífices del darwinismo estadístico fueron principalmente Haldane, Wright, Chetverikov y Fisher. En este período, el conflicto entre el carácter discreto de la herencia y el gradualismo9 se resuelve mediante una fusión de perspectivas: los métodos experimentales de la genética mendeliana y los métodos estadísticos, que empleados antes por Galton y los biométricos para estudiar los fenotipos se aplican ahora a los genes. Los efectos de la selección natural se miden estudiando variaciones de frecuencias genéticas en poblaciones, la evolución resulta de la acumulación de pequeños cambios del material genético, y las nuevas variantes, una vez introducidas, acaban fijándose en las poblaciones. Esta solución preserva el gradualismo al tiempo que hace de la selección una fuerza creativa, ya que las variaciones no se consideran sólo dañinas, sino que proporcionan a la selección natural un gradiente en el que actuar. Al menos en la versión americana de Wright, menos «seleccionista» que la de Fisher, la selección natural debe actuar en colaboración con la deriva genética, pues sólo ésta permite abandonar óptimos locales y lograr posiciones adaptativas de otro modo inaccesibles.

Finalmente, la etapa más reciente del darwinismo inaugura una perspectiva en construcción que consideraría a la selección natural como una parte intrín-

9 Hugo de Vries, Carl Correns y Erick von Tschermak, los primeros continuadores del redescubierto trabajo de Mendel, no aceptaban la teoría de la selección natural, pues la herencia mendeliana les proveía de un mecanismo de especiación saltacionista por el que la evolución se explicaba únicamente en términos de herencia genética y mutación. (Cfr. W. B. Provine, 1971). 
seca de un proceso complejo, dinámico y termodinámico. Desde este punto de vista, la selección natural habría evolucionado, ella misma, en una subclase de sistemas auto-organizativos que habrían adquirido la habilidad de retener y trasmitir la información que facilita su propia reproducción. Los trabajos de Kauffman sobre modelización de procesos evolutivos complejos mediante redes booleanas aleatorias, donde gran parte del orden inherente proviene de la propia dinámica auto-organizativa del sistema, constituyen un ejemplo paradigmático de este nuevo episodio en la historia del darwinismo.

Aunque en 1995 Depew y Weber consideraron que las aportaciones más interesantes para caracterizar el darwinismo autoorganizativo procedían del campo de los sistemas complejos (Solé 2009), hoy día habría que ampliar el espectro de las aportaciones a una perspectiva que verdaderamente permita incorporar la teleología orgánica a las teorías biológicas. En este sentido, creemos necesario incluir el trabajo realizado dentro de la biología evolutiva del desarrollo ${ }^{10}$, así como el de la biología de sistemas, que está permitiendo superar parcialmente el reduccionismo inherente a la biología molecular (Etxeberria y Umerez 2006). Por otro lado, los trabajos en la frontera entre lo inanimado (lo todavía no vivo) y lo propiamente vivo, como las investigaciones en el campo del origen de la vida, la biología sintética o la vida artificial, permiten replantear la cuestión de la definición de vida en base a modelos y experimentos de sistemas intermedios o transeúntes (ver Etxeberria y Ruiz-Mirazo 2009). Estos nuevos enfoques permen avanzar en el trabajo tanto científico como filosófico del problema de la singularidad de los organismos, tal y como fue planteado por Kant.

\section{Más allá de Kant: El problema de la función y de la clasificación hoy}

Como hemos visto, para Kant la física newtoniana no era sólo un esquema coherente para ordenar y predecir los fenómenos, sino que proporcionaba un modelo para entender cómo es posible conocer la naturaleza: las formas a prio$r i$ de la Razón que moldean nuestra experiencia asumen la verdad de la física newtoniana. Sin embargo, los problemas planteados por el desarrollo de las geo-

${ }^{10}$ Ver Etxeberria y Nuño de la Rosa 2009a para un análisis del trabajo pionero de Pere Alberch en esa corriente. 
metrías no euclidianas y la teoría de la relatividad a fines del XIX y principios del XX motivaron la búsqueda de otras vías en el neokantismo. Recogiendo algunas de estas propuestas, sobre todo las de Cassirer, Friedman ha considerado recientemente que el juicio sintético a priori de Kant debe ser corregido por uno relativizado e histórico $(2001,2004)^{11}$.

Por tanto, en la medida en que la ciencia newtoniana puede considerarse actualmente «superada», los proyectos de naturalización no pueden, como en tiempos de Kant, remitirse a aquella, sino que la «filosofía científica» debería estar "profundamente comprometida» con los resultados ofrecidos por la mejor ciencia actual (Friedman 2004, p. 94). Hemos visto que autores como Depew y Weber creen que esto es, precisamente, lo que ha sucedido en la historia de la teoría evolutiva, pues consideran que la versión «más evolucionada» del darwinismo descansa en la consideración de las propiedades autoorganizativas de los seres vivos y no renuncia, por tanto, a afirmar la irreductibilidad de lo vivo frente a lo inanimado. Winther (2008) nos ofrece un retrato similar del darwinismo, confiando en que la pluralidad de visiones no se agote en dicotomías enfrentadas ${ }^{12}$, sino que abra un espacio multidimensional e integrativo para la biología. Aunque la naturalización de la explicación de los fenómenos vivientes sigue siendo un problema absorbente para la filosofía de la biología, el propio desarrollo del darwinismo muestra que el ideal naturalizador está en permanente transformación, y que, desde luego, hace tiempo que ha dejado atrás el paradigma newtoniano como modelo.

Como vimos en la segunda sección, Kant considera que los organismos están formados por partes que han sido conformadas por otras partes y que son, por tanto, causa y efecto de ellas (en ese sentido las partes dependen del todo).

La filosofía de la biología estándar ha aceptado que la solución para naturalizar la teleología es la concepción etiológica, que define la función de una par-

11 «Yo mismo creo que un descendiente del original sintético a priori de Kant —un descendiente relativizado, historizado y dinámico- es lo que emerge más claramente en nuestra situación conceptual actual» (Friedman 2004, pp. 95-96).

12 Winther (2008) considera tres dimensiones del darwinismo: su patrón explicativo (sean las leyes o la narrativa), el nivel de selección (sea el organismo o pluralista, jerárquico) y el grado de diferencia entre las unidades del mismo tipo (que caracteriza las diferencias entre el pensamiento variacional y el esencialista). 
te en términos de su historia selectiva: la función de algo es aquello que hizo que resultara elegido por la selección natural entre las variantes entonces existentes ${ }^{13}$. Desde esta perspectiva, tanto las funciones orgánicas como las relaciones entre los planes corporales y las partes orgánicas (homologías) pueden naturalizarse atendiendo a la historia selectiva de los organismos. El problema es que esta solución, si bien elimina en parte la teleología, también asimila los seres vivos a los artefactos, a las entidades creadas con un fin o propósito externo a ellos.

Recuperando la definición kantiana de teleología, se ha objetado que la noción etiológica de función no entiende la función en el contexto de la organización de las partes que la instancian y no puede dar cuenta de la autorregulación actual de los sistemas orgánicos. Se trata de un concepto aséptico que los simpatizantes de la noción de organismo como fin natural no pueden aceptar filosóficamente. En terminología actual, señalar que cada organismo muestra una teleología interna significa afirmar que: 1) son sistemas regulados por una causalidad circular cuyo fin es la supervivencia o propagación del sistema; 2) el sistema es el mismo si consiste en partes diferentes en tiempos diferentes, lo que implica que debe haber existido previamente a sus partes (McLaughlin, 2001). Por tanto, la teleología es aquí una cuestión ontológica y conlleva una vuelta a explorar la organización interna de los organismos y su desarrollo desde la perspectiva de fisiología o la morfología, más que desde su origen histórico (Lewens 2007).

Para ilustrar el problema de las relaciones morfológicas revisaremos la noción de homología y el diferente papel que juega en biología dependiendo del punto de vista teórico desde el que se analiza, en este caso, la Síntesis Moderna y la Biología evolutiva del desarrollo (Etxeberria y Nuño de la Rosa 2009b).

El concepto de homología se refiere a la mismidad subyacente a las distintas partes del cuerpo u otros rasgos orgánicos. Su definición ha cambiado en el curso de la historia de la biología, y en la teoría de la evolución actual coexisten enfoques muy distintos del problema de la identificación y la explicación de las par-

13 Hay que tener en cuenta que si decimos que un rasgo ha sido seleccionado, su elucidación no puede dejar de tener en cuenta qué variantes existían en el momento de la selección, de forma que la variedad elegida es la mejor de entre las existentes en ese momento (y bajo circunstancias determinadas). De otro modo: la función etiológica no admite razonamientos en términos de contrafácticos sin caer en una tautología. 
tes homólogas (Brigandt 2002). Como vimos arriba, el concepto filogenético de homología considera que dos caracteres son homólogos cuando se derivan de un rasgo equivalente en el ancestro común. De este modo, las homologías se distinguen de las analogías, definidas como convergencias adaptativas producidas por la selección natural ante parecidas demandas ambientales. Esta definición de homología encaja con los objetivos de la sistemática, dedicada a identificar sinapomorfías, es decir, semejanzas orgánicas que, a través de su congruencia con datos sobre otros caracteres, permiten rastrear filogenias para, en último término, ordenar el reino orgánico mediante árboles filogenéticos verosímiles. Esta línea de investigación no afirma nada sobre la naturaleza de las partes estudiadas, ya que el objetivo último es filogenético o sistemático.

Sin embargo, la evo-devo ha mostrado un interés por los caracteres homólogos que no es meramente clasificatorio. Asumiendo la crítica de Gould y Lewontin al atomismo adaptacionista, el concepto ontogenético de homología se propone como objetivo la búsqueda de formas «naturales» de reconocer las partes orgánicas, recuperando el proyecto de la morfología trascendental. La gran diferencia con respecto a aquella radica en que la evo-devo pretende explicar causalmente (es decir, ontogenéticamente) las homologías. Desde esta perspectiva, las semejanzas morfológicas pueden comprenderse en términos de procesos generativos compartidos, y la variedad, en términos de sus transformaciones. Así, Wagner (1994) considera que las partes fenotípicas se individualizan durante el desarrollo, siendo homólogas «si comparten un conjunto de constricciones ontogenéticas». Ese conjunto de constricciones permite comprender las homologías mediante la definición de un espacio paramétrico de variedad ${ }^{14}$, de modo que las novedades evolutivas pueden explicarse, dentro de la misma lógica, como resultado de la ruptura de las constricciones y la irrupción de otras nuevas que, a su vez, definirían un nuevo espacio paramétrico de variedad.

En este nuevo marco teórico, las homologías pueden volver a definirse no sólo por su ascendencia común sino en términos estructurales. Como clase natural, una homología puede definirse como una agrupación de partes de organis-

${ }_{14}$ Puesto que los mismos procesos ontogenéticos pueden estar regulados por genes distintos, los factores que determinan los parámetros de variación se definen a escala epigenética: la tasa de división celular, el nivel de adhesión celular, la zona de contacto entre dos tejidos embrionarios, etc. 
mos que comparten semejanzas estables, resultado de constricciones del desarrollo también comunes.

Así, la biología evolutiva del desarrollo puede reivindicar a la morfología trascendental como predecesora. La evo-devo arranca precisamente de la constatación del carácter discreto del morfoespacio, reconociéndolo como una variedad «dada», pero la explica atendiendo a causas mecánicas, a saber, las constricciones del desarrollo. Al mismo tiempo, la función de las partes no puede estudiarse atendiendo sólo a la ventaja adaptativa que confieren al organismo, pues esta ventaja ya no puede explicar la presencia de la parte, que habrá de estudiarse como una homología ontogenética (Griffiths 2006). Por ello, creemos que esta concepción de las partes de los organismos y de sus funciones permitiría aunar dos perspectivas de la vida a veces disonantes en las diferentes disciplinas biológicas, la evolutiva y la organizacional, en el marco de un nuevo darwinismo.

\section{Conclusiones}

A lo largo de este artículo hemos examinado la herencia de Kant en la obra de Darwin y en las tradiciones darwinistas posteriores desde el punto de vista de su concepción de ciencia y de organismo. En la biología decimonónica, la influencia de la filosofía kantiana se bifurca en dos direcciones que tendrán consecuencias muy distintas en biología: por un lado, el idealismo romántico en el que arraigan las investigaciones morfológicas de la Naturphilosophie y, por otro, el mecanicismo en el que se consolida el neokantismo, y que da lugar a la teoría darwiniana de la evolución. Aunque Kant pensó que sólo el paradigma mecanicista newtoniano permitiría una ciencia de los organismos, desde entonces las propias ciencias ha proporcionado modelos más adecuados para explicar los seres vivos ${ }^{15}$. De ahí que se revele fundamental preguntarnos, desde el presente, si tales modelos sustentan la concepción kantiana de la vida y en qué sentido ese apoyo puede considerarse un avance para la biología y la filosofía contemporáneas en relación a las dos cuestiones esenciales que venimos analizando: la relación entre teleología y mecanismo y el problema del orden taxonómico y su carácter histórico.

15 Así dicen Weber y Varela: «El verdadero «Newton de la brizna de hierba» no fue un individuo, sino una convergencia histórica de pensamiento filosófico y biológico en un caldero solidario" (2002, p. 121). Grene y Depew (2004) manifiestan una posición parecida. 
A la luz de los desarrollos en biología del desarrollo y evo-devo, las opiniones al respecto del lugar que debe ocupar en la filosofía de la biología actual el análisis kantiano de la relación entre mecanicismo y teleología son divergentes. Algunos autores defienden la recuperación de la teleología en un sentido estrictamente kantiano. Así, Lewens (2007) considera que los organismos han de volver a considerarse (regulativamente) fines naturales, pues —en su opinión- la finalidad biológica continúa inaprensible a la formalización científica. En el extremo opuesto, se ha dicho que Kant tiene poco que ofrecer al naturalismo, que habría de inspirarse en filosofías que afronten el problema de la naturaleza orgánica desde una perspectiva ontológica (y no epistemológica). Es el caso de Zammito (2006), quien cree que la vuelta a la distinción aristotélica entre causa material y formal o a la discriminación de Locke entre partes y partículas, donde la «teleología intrínseca» se considera ontológicamente, resultaría mucho más provechosa para una reconceptualización de la investigación biológica de los rasgos asociados a la autorregulación. Coincidimos con Zammito en la necesidad de recuperar la reflexión ontológica sobre los organismos, pero, a su vez, creemos también que las herramientas matemáticas desarrolladas en el último siglo para modelizar los sistemas complejos permiten replantear el concepto de intuición (Pérez Herranz 1998) y legitimar así, epistemológicamente, una nueva ciencia de los organismos.

Con respecto al problema del orden taxonómico, consideramos que la cladística ofrece hoy el mejor método para la clasificación de los seres vivos. Sin embargo, creemos también que la taxonomía no agota el estudio de las «afinidades morfológicas» que, como señalara Kant, parecen presentársenos fenoménicamente. Como hemos visto, la morfología evolucionista y la biología evolutiva del desarrollo han permitido replantear esta cuestión desde una perspectiva causal, lo que ha posibilitado a la filosofía concebir a las homologías ontogenéticas y los planes corporales como clases naturales. La investigación de las formas que han permanecido estables en el tiempo, las mismas que fueron objeto de la morfología trascendental, sigue siendo, por tanto, un objetivo ineludible para un proyecto de corte kantiano que estudie la historia de la vida desde un punto de vista radicalmente apegado a las formas históricas empíricas. Por otro lado, el problema del origen de la vida, central para configurar la postura de Kant, sigue constituyendo el mayor desafío para la visión darwiniana del árbol de la vida, dada la creciente evidencia de que en las etapas más tempranas, la evolución microbiana no se produjo por ramificación, sino, fundamentalmente, por asociación (Margulis 1998). 
En definitiva, Kant continúa siendo una fuente de inspiración para la filosofía de la biología. Su planteamiento de la fenomenología de lo vivo sigue vigente, a la vez que ciertos programas de investigación fundamentan el desarrollo de nuevas respuestas a los problemas que nos legó. Darwin pudo ser el Newton de la brizna de hierba, pero la filosofía de la biología actual no necesita un Newton, sino un nuevo darwinismo sistémico.

\section{Agradecimientos}

AE agradece financiación de los proyectos IT-250-07 (Gobierno Vasco) y FFI2008-06348-C01/02/FISO (MICINN). LNR agradece la financiación proporcionada por un contrato de investigación predoctoral de la Universidad Complutense de Madrid.

\section{Bibliografía}

Albarracín, A., 1983. La teoría celular, historia de un paradigma, Madrid: Alianza Universidad.

Álvarez, J.R., 1998. «Una débil esperanza: La idea kantiana de una ciencia biológica». Themata 20: 49-66.

Amundson, R., 2005. The Changing Role of the Embryo in Evolutionary Thought: Roots of Evo-Devo, Cambridge MA: Cambridge University Press.

Ariew, A., Cummins, R. \& Perlman M., 2002. Functions. New Essays in the Philosophy of Psychology and Biology, Oxford: Oxford University Press.

BlanchetTe, R., 2002., Le problème de la classification en zoologie, Tesis doctoral dirigida por MURRAY, W., Université Laval.

Bortoft, H., «Prólogo». En von Goethe, J.W., 2002. Goethe y la ciencia, J. Naydler, J. ed., Madrid: Siruela.

BOWLER, P. J., Charles Darwin: El hombre y su influencia (Trad. Eloy Rada García), Madrid: Alianza, 1995.

BRIGANDT, I., 2002. Homology and the origin of correspondence, Biology and Philosophy 17: 389-407. 
CAssirer, E., 1974. Kant, vida y doctrina, México D.F.: Fondo de Cultura Económica, 2. ${ }^{\mathrm{a}} \mathrm{ed}$.

DARWIN, C. El origen de las especies, 6. ${ }^{a}$ ed., (trad. Antonio de Zulueta), Madrid: Alianza, 2003.

DaWKINS, R., 1976. El gen egoísta. Las bases biológicas de nuestra cultura, Barcelona: Salvat, 2002.

DePew, D.J. y WeBer, B.H., 1995. Darwinism evolving. Systems Dynamics and the Genealogy of Natural Selection, Cambridge (MA) y Londres: MIT Press.

ETXEBERRIA, A. y NuÑo DE LA RoSA, L., 2009a. "A world of opportunity within constraint: Pere Alberch's early evo-devo». En D. Rasskin-Gutman and M. De Renzi (eds.) Pere Alberch. The creative trajectory of an evo-devo biologist, Valencia: Publications Universitat de València. En prensa.

- 2009b. «Partes y funciones en el desarrollo y la evolución. Hacia un darwinismo sistémico». En H. Dopazo \& A. Navarro Eds., Evolución y Adaptación: 150 años después del Origen de las Especies, Madrid: SESBE. En prensa.

Etxeberria, A. \& Ruiz-Mirazo, K., 2009. The challenging biology of transientes. A view from the perspective of autonomy, EMBO Reports 10: S33-S36.

ETXEBERRIA. A. y UMEREZ, J., 2006. «Organización y organismo en la Biología Teórica ¿Vuelta al organicismo?», Ludus Vitalis 26: 3-38.

FISHER, R. A., 1959. «Natural selection from the genetical standpoint», Australian Journal of Science 22: 16-17.

Friedman, M., 2001. Dynamics of reason, Stanford: CSLI Publications.

— 2004. «Philosophy as dynamic reason. The idea of scientific philosophy». En H. Carel y D. Gamez Eds. What philosophy is, Manchester: Continuum, pp. 73-96.

GARCía AZKONOBIETA, T., 2005. «Evolución, desarrollo y (auto) organización. Un estudio sobre los principios filosóficos de la evo-devo.» Tesis doctoral, Universidad del País Vasco, accesible en http://www.ehu.es/ias-research/garcia/TESIS.pdf

GAYON, J., 1998. «La marginalisation de la forme dans la biologie de l'évolution», Bulletin de la Société d'histoire et d'épistémologie des sciences de la vie 5 (2): 133-166.

— 2003. «From Darwin to today in evolutionary biology». En J. Hodge and J. Radick Eds. The Cambridge Companion to Darwin, Cambridge, RU: Cambridge University Press, pp. 240-264.

GILBERT, S., 2003. «The morphogenesis of evolutionary developmental biology», International Journal of Developmental Biology 47 (7/8): 467-478. 
Ginsborg, H., (2008). Kant's Aesthetics and Teleology. En The Stanford Encyclopedia of Philosophy (Fall 2008 Edition), Edward N. Zalta (ed.).

Ginzburg, L. R. y M. Colyvan., 2004. Ecological Orbits: How Planets Move and Populations Grow, Oxford University Press, USA.

GonzÁlez Recio, J. L., 2004. Teorías de la vida, Madrid: Síntesis.

Gould S. J., 1983. «The hardening of the modern synthesis». En Grene M (Ed.) Dimensions of Darwinism, Cambridge: Cambridge University Press, pp. 71-93.

- 2002/2004. La Estructura de la Teoría de la Evolución, Barcelona: Tusquets.

Gould JS, y LeWontin, R., 1979. The Spandrels of San Marco and the Panglossian Paradigm: A Critique of The Adaptationist Programme, Proceedings Of The Royal Society of London, Series B 205 (1161): 581-598.

Grene, M. G. y DePew, D. J. 2004. The philosophy of biology: an episodic history, Cambridge: Cambridge University Press.

Griffiths, P.E. 2006. Function, Homology and Character Individuation, Philosophy of Science, 73(1): 1-25.

HunEman, P., 2006. «Naturalising purpose: From comparative anatomy to the "adventure of reason" ", Studies in History and Philosophy of Biol \& Biomed Sci 37, no. 4: 649-674.

Huneman, P. Ed. 2007. Understanding purpose: Kant and the Philosophy of Biology, Rochester, NY: University of Rochester Press.

INSUA RODRíGUEZ, P., 2005. «Biología e individuo corpóreo: el problema del «sexto predicable» 1. Sentido darvinista de la evolución», El Catoblepas, 41.

Kant, I. Crítica del juicio, (Traductor: MG García Morente) México D.F.: Porrúa, $1790 / 1973$.

KANT, I., 2004. «Sobre el uso de los principios teleológicos en la filosofía», Logos. Anales del Seminario de Metafisica (Trad. N. Sánchez Madrid) 7-47.

LAMARCK, J. B. M., 1873. Philosophie zoologique, ou Exposition des considérations relatives à l'histoire naturelle des animaux, Paris: F. Savy.

Lewens, T., 2007. «Functions». En M. Matthen y C. Stephens Eds. Philosophy of Biology, Amsterdam: Elsevier, pp. 525-547.

MaYr, E., 1982. The Growth of Biological Thought: Diversity, Evolution, and Inheritance, Cambridge MA: The Belknap Press of Harvard University Press.

- 1992. Una larga controversia: Darwin y el darwinismo, Madrid: Crítica. 
MARGUlis, L, 1998. Symbiotic planet: a new view of evolution, New York: Basic Books.

MCLAUGHLin, P., 2001. What functions explain: Functional explanation and self-reproducing systems, Cambridge: Cambridge University Press.

Pérez Herranz, F. M., 1998. Arthra Hê Péphiken. Las articulaciones naturales de la Filosofia, Alicante: Publicaciones de la Universidad de Alicante.

Provine, W. B., 1971. The origins of theoretical population genetics, Chicago: The University of Chicago Press.

RÁDL, E., 1988. Historia de las teorías biológicas, Madrid: Alianza.

RUSE, M., 1975. «Darwin's debt to philosophy: an examination of the influence of the philosophical ideas of John FW Herschel and William Whewell on the development of Charles Darwin's theory of evolution», Stud Hist Philos Sci, 6(2): 159-81.

- 1983. La revolución darwinista: la ciencia al rojo vivo (Trad. C. Castrodeza), Madrid: Alianza.

SLOAN, P.R., 2001. «Historia natural 1670-1802». En A. Barahona, E. Suarez, S. Martínez Eds. Filosofía e Historia de la Biología. México: UNAM, pp. 41-64.

SOLÉ, R., 2009. Redes complejas. Del genoma a Internet, Barcelona: Tusquets.

WAGNER, G.P., 1994. «Homology and the Mechanisms of Development». En B.K. Hall (ed.), Homology: The Hierarchical Basis of Comparative Biology, San Diego: Academic Press, pp. 273-299.

WALSH, D., 2006. «Organisms as natural purposes: The contemporary evolutionary perspective», Studies in History and Philosophy of Biol \& Biomed Sci 37 (4): 771-791.

Wouters, A., 2005. «The Function Debate in Philosophy», Acta Biotheoretica 53(2): 123-151.

Weber, A. y VARelA, FJ., 2002. «Life after Kant: Natural purposes and the autopoietic foundations of biological individuality», Phenomenology and the Cognitive Sciences 1: 97-125.

Winther RG., 2008. Systemic Darwinism, PNAS 105 (33): 11833-11838.

ZAMmiTO, J., 2006. «Teleology then and now: The question of Kant's relevance for contemporary controversies over function in biology,» Studies in History and Philosophy of Biol \& Biomed Sci 37 (4): 748-770.

Recibido: 11/10/2009

Revisado: 21/10/2009 